\title{
Bose-Einstein condensates of atoms with arbitrary spin
}

\author{
P Van Isacker $\dagger$ and S Heinze \\ † Grand Accélérateur National d'Ions Lourds, CEA/DSM-CNRS/IN2P3, BP 55027, \\ F-14076 Caen Cedex 5, France \\ $\ddagger$ Institute of Nuclear Physics, University of Cologne, Zülpicherstrasse 77, 50937 \\ Cologne, Germany
}

\begin{abstract}
We show that the ground state of a Bose-Einstein condensate of atoms with hyperfine spin $f=2$ can be either spin aligned, condensed into pairs of atoms coupled to $F=0$, or condensed into triplets of atoms coupled to $F=0$. The complete phase diagram is constructed for $f=2$ and the generic properties of the phase diagram are obtained for $f>2$.
\end{abstract}

PACS numbers: 03.75.Fi, 03.65.Fd

If atoms in a Bose-Einstein condensate (BEC) are trapped by optical means [1], their hyperfine spins (or spins) are not frozen in one particular direction but are essentially free but for their mutual interactions. As a result, the atoms do not behave as scalar particles but each of the components of the spin is involved in the formation of the BEC. This raises interesting questions concerning the structure of the condensate and how it depends on the spin exchange interactions between the atoms.

Such questions were addressed in a series of theoretical papers by Ho and coworkers [2] who obtained solutions based on a generating function method. In the case of spin-1 atoms the problem of quantum spin mixing was analyzed by Law et al. [3] who proposed an elegant solution based on algebraic methods. It is the purpose of this paper to point out that a wide class of many-body hamiltonians appropriate for the problem of interacting bosons with spin can be solved through algebraic techniques which have found fruitful applications in nuclear physics [4] as well as in other fields of physics (see, e.g. Ref. [5]). The main result derived in this paper is that an exact solution is available for spin values $f=1$ and $f=2$ (for any value of the number of atoms $N$ ) which allows the analytic determination of the structure of the ground state of the condensate. For spin values $f>2$ solvable classes of hamiltonians give insights into the generic properties of the phase diagram.

We consider a one-component dilute gas of trapped bosonic atoms with arbitrary (integer) hyperfine spin $f$. In second quantization the hamiltonian of this system has a one-body and a two-body piece that can be written as (we follow the notation of 
Ref. [3])

$$
\begin{aligned}
\mathcal{H} \equiv & \mathcal{H}_{1}+\mathcal{H}_{2}=\sum_{m} \int \hat{\Psi}_{m}^{\dagger}\left(-\frac{\nabla^{2}}{2 M_{\mathrm{a}}}+V_{\text {trap }}\right) \hat{\Psi}_{m} d^{3} x \\
& +\sum_{m_{i}} \Omega_{m_{1} m_{2} m_{3} m_{4}} \int \hat{\Psi}_{m_{1}}^{\dagger} \hat{\Psi}_{m_{2}}^{\dagger} \hat{\Psi}_{m_{3}} \hat{\Psi}_{m_{4}} d^{3} x
\end{aligned}
$$

where $\hbar=1, M_{\mathrm{a}}$ is the mass of the atom, and $\hat{\Psi}_{m}$ and $\hat{\Psi}_{m}^{\dagger}$ are the atomic field annihilation and creation operators associated with atoms in the hyperfine state $|f m\rangle$ with $m=-f, \ldots,+f$, the possible values of all summation indices in (11). The trapping potential $V_{\text {trap }}$ is assumed to be the same for all $2 f+1$ components. The coefficients $\Omega_{m_{1} m_{2} m_{3} m_{4}}$ follow from the interaction between atoms which is assumed to be of shortrange, two-body character,

$$
U\left(\vec{x}_{i}, \vec{x}_{j}\right)=\delta\left(\vec{x}_{i}-\vec{x}_{j}\right) \sum_{F M} \nu_{F}^{\prime}\left|f^{2} ; F M\right\rangle\left\langle f^{2} ; F M\right|,
$$

where $\left|f^{2} ; F M\right\rangle$ is the combined state of the atoms $i$ and $j$ with total spin $F$, and $\nu_{F}^{\prime} \equiv 4 \pi \hbar^{2} a_{F} / M_{\mathrm{a}}$ with $a_{F}$ being the $s$-wave scattering length in the $F$ channel. The assumption underpinning the form (2) is rotational invariance of the hamiltonian in hyperfine-spin space.

We assume in this paper that the scattering lengths in the different $F$ channels are comparable and that, in first approximation, the interaction strength between the bosons is independent of $F$. In that case the dominant part of the hamiltonian (1) is of the form

$$
\mathcal{H}_{\mathrm{s}}=\mathcal{H}_{1}+\lambda^{\prime} \sum_{m_{1} m_{2}} \int \hat{\Psi}_{m_{1}}^{\dagger} \hat{\Psi}_{m_{2}}^{\dagger} \hat{\Psi}_{m_{1}} \hat{\Psi}_{m_{2}} d^{3} x
$$

and is symmetric under any interchange of the spin-component indices. Under this assumption the condensate wave functions for each spin component $\phi_{m}(\vec{x})(m=$ $-f, \ldots,+f)$ can be approximated by a single wave function $\phi(\vec{x})$ which satisfies the Gross-Pitaevskii equation associated with the dominant hamiltonian [3]. Furthermore, the atomic field creation and annihilation operators at zero temperature can be approximated by

$$
\hat{\Psi}_{m}^{\dagger} \approx b_{m}^{\dagger} \phi(\vec{x}), \quad \hat{\Psi}_{m} \approx b_{m} \phi(\vec{x}), \quad m=-f, \ldots,+f,
$$

where $b_{m}$ and $b_{m}^{\dagger}$ are annihilation and creation operators associated with the entire condensate, satisfying the usual boson commutation rules

$$
\left[b_{m}, b_{m^{\prime}}^{\dagger}\right]=\delta_{m m^{\prime}}, \quad\left[b_{m}, b_{m^{\prime}}\right]=\left[b_{m}^{\dagger}, b_{m^{\prime}}^{\dagger}\right]=0 .
$$

In this approximation the entire hamiltonian (1) can be rewritten as

$$
\mathcal{H} \approx \hat{H} \equiv \epsilon b^{\dagger} \cdot \tilde{b}+\frac{1}{2} \sum_{F} \nu_{F}\left[b^{\dagger} \times b^{\dagger}\right]^{(F)} \cdot[\tilde{b} \times \tilde{b}]^{(F)},
$$

where the coefficients $\epsilon$ and $\nu_{F}$ are related to those in the original hamiltonian through integration over $x$, viz. $\nu_{F}=\nu_{F}^{\prime} \int|\phi(\vec{x})|^{4} d^{3} x$. The notation $\times$ in Eq. (6) implies the coupling to a given spin $F$ and projection $M$,

$$
\left[b^{\dagger} \times b^{\dagger}\right]_{M}^{(F)}=\sum_{m m^{\prime}}\left\langle f m f m^{\prime} \mid F M\right\rangle b_{m}^{\dagger} b_{m^{\prime}}^{\dagger},
$$


where $\langle\cdots \cdots\rangle$ is a Clebsch-Gordan coefficient [6]. Furthermore, the dot $\cdot$ denotes a scalar product,

$$
\hat{T}^{F} \cdot \hat{T}^{F} \equiv(-)^{F} \sqrt{2 F+1}\left[\hat{T}^{F} \times \hat{T}^{F}\right]_{0}^{(0)},
$$

for tensor operators $\hat{T}_{M}^{F}$ of rank $F$. The definition of the adjoint operator $\tilde{b}_{m} \equiv$ $(-)^{f-m} b_{-m}$ ensures that $\tilde{b}_{m}$ is an annihilation operator with transformation properties under rotations that are the same as those for the creation operator $b_{m}^{\dagger}$ [7]. With the above definitions we have that $b^{\dagger} \cdot \tilde{b}=\sum_{m} b_{m}^{\dagger} b_{m}$ is the number operator $\hat{N}$ which counts the total number of atoms in the condensate.

To derive the solvability properties of the hamiltonian (6), we first determine its algebraic structure by introducing the bilinear operators $b_{m}^{\dagger} b_{m^{\prime}}$. From Eq. (5) one finds the commutation relations

$$
\left[b_{m_{1}} b_{m_{2}}^{\dagger}, b_{m_{3}} b_{m_{4}}^{\dagger}\right]=b_{m_{1}} b_{m_{4}}^{\dagger} \delta_{m_{2} m_{3}}-b_{m_{3}} b_{m_{2}}^{\dagger} \delta_{m_{1} m_{4}}
$$

which can be identified as those of the unitary (Lie) algebra $\mathrm{U}(2 f+1)$ [7]. Exactly solvable hamiltonians with rotational or $\mathrm{SO}(3)$ invariance are now found by the determination of all Lie algebras $G$ satisfying $\mathrm{U}(2 f+1) \supset G \supset \mathrm{SO}(3)$. The canonical reduction of $\mathrm{U}(2 f+1)$ is of the form

$$
\mathrm{U}(2 f+1) \supset \mathrm{SO}(2 f+1) \supset \mathrm{SO}(3) .
$$

[For $f=3$ there is an additional exceptional $\mathrm{G}_{2}$ algebra between $\mathrm{SO}(2 f+1)$ and $\mathrm{SO}(3)$ which for the symmetric representations of $\mathrm{U}(2 f+1)$ considered here does not add anything to the discussion.] The relevance of a chain of nested algebras of the type (10) is that it defines a set of commuting operators and with it a class of solvable hamiltonians. Consider in particular the hamiltonian

$$
\begin{aligned}
\hat{H}^{\prime} & =a_{1} \hat{C}_{1}[\mathrm{U}(2 f+1)]+a_{2} \hat{C}_{2}[\mathrm{U}(2 f+1)] \\
& +b \hat{C}_{2}[\mathrm{SO}(2 f+1)]+c \hat{C}_{2}[\mathrm{SO}(3)],
\end{aligned}
$$

where $a_{1}, a_{2}, b$, and $c$ are numerical coefficients and $\hat{C}_{n}[G]$ is the $n^{\text {th }}$-order Casimir operator of the algebra $G$ which satisfies the property that it commutes with all generators of $G$ [8]. Solvability of the hamiltonian (11) follows from the fact that it is written as a sum of commuting operators, a property which indeed is valid for the Casimir operators associated to any chain of nested algebras such as (10). The Casimir operators appearing in Eq. (11) are known in closed form,

$$
\begin{aligned}
& \hat{C}_{1}[\mathrm{U}(2 f+1)]=\hat{N} \\
& \hat{C}_{2}[\mathrm{U}(2 f+1)]=\hat{N}(\hat{N}+2 f), \\
& \hat{C}_{2}[\mathrm{SO}(2 f+1)]=-(2 f+1) \hat{T}_{+}^{0} \cdot \hat{T}_{-}^{0}+\hat{N}(\hat{N}+2 f-1), \\
& \hat{C}_{2}[\mathrm{SO}(3)]=\sum_{F}\left[\frac{1}{2} F(F+1)-f(f+1)\right] \hat{T}_{+}^{F} \cdot \hat{T}_{-}^{F}+f(f+1) \hat{N},
\end{aligned}
$$

in terms of the operators $\hat{T}_{+, M}^{F} \equiv\left[b^{\dagger} \times b^{\dagger}\right]_{M}^{(F)}$ and $\hat{T}_{-, M}^{F} \equiv[\tilde{b} \times \tilde{b}]_{M}^{(F)}$. Equations (12) show that the solvable hamiltonian (11) is a special case of the general hamiltonian (6) with 
coefficients $\epsilon$ and $\nu_{F}$ that are linear combinations of $a_{1}, a_{2}, b$, and $c$ according to

$$
\begin{aligned}
& \epsilon=a_{1}+(2 f+1) a_{2}+2 f b+f(f+1) c, \\
& \nu_{F}=2 a_{2}+2 b+[F(F+1)-2 f(f+1)] c, \quad F \neq 0, \\
& \nu_{0}=2 a_{2}-4 f b-2 f(f+1) c,
\end{aligned}
$$

The eigenvalues of the hamiltonian (11) are

$$
E^{\prime}(N, v, F)=a_{1} N+a_{2} N(N+2 f)+b v(v+2 f-1)+c F(F+1) .
$$

The allowed values of $v$ are $v=N, N-2, \ldots, 1$ or 0 , as can be obtained from the $\mathrm{U}(2 f+1) \supset \mathrm{SO}(2 f+1)$ branching rule [8]. The quantum number $v$ corresponds to the number of bosons not in pairs of bosons coupled to $F=0$, and is known as seniority [9, 10]. The allowed values of the total spin $F$ are obtained from the $\mathrm{SO}(2 f+1) \supset \mathrm{SO}(3)$ branching rule which is rather complicated but known in general [11]. The $f=2$ example is discussed below.

The generic solvability properties of the original hamiltonian (6) now follow from a simple counting argument. For atoms with spin $f=1$ the solvable hamiltonian (11) has three coefficients $a_{1}, a_{2}$, and $c$ [since $\left.\mathrm{SO}(2 f+1)=\mathrm{SO}(3)\right]$ while the general hamiltonian (6) also contains three coefficients $\epsilon, \nu_{0}$, and $\nu_{2}$. [Note that the coupling of two spins to odd $F$ is not allowed in the approximation (44) of a common spatial wave function, so no $\nu_{1}$ term occurs.] For atoms with spin $f=2$ both the solvable and general hamiltonian contains four coefficients $\left(a_{1}, a_{2}, b\right.$, and c versus $\epsilon, \nu_{0}, \nu_{2}$, and $\left.\nu_{4}\right)$ which can be put into one-to-one correspondence. Hence the general hamiltonian (6) is solvable for $f=2$. The same counting argument shows that it is no longer solvable for $f>2$.

The case of interacting $f=1$ atoms was discussed by Law et al. [3] who identified the existence of two possible condensate ground states: one with all atoms aligned to maximum spin $F=N$ and a second with pairs of atoms coupled to $F=0$. Whether the condensate is aligned or paired depends on a single interaction parameter which in our notation is $c$. With the technique explained above we can also derive the phase diagram for atoms with spin $f=2$. The results are exact and valid for arbitrary $N$. The entire spectrum is determined by the eigenvalue expression (14) together with the necessary branching rules. In particular, the allowed values of total spin $F$ for a given seniority $v$ are derived from the $\mathrm{SO}(5) \supset \mathrm{SO}(3)$ branching rule [4] given by $F=2 \tau, 2 \tau-2,2 \tau-3, \ldots, \tau+1, \tau$ with $\tau=v, v-3, v-6, \ldots$ and $\tau \geq 0$.

It is now possible to determine all possible ground-state configurations of the condensate. This problem has been considered in the study of the spectral features of quantal systems with random interactions [12]. We note that the character of the ground state does not depend on the coefficients $a_{i}$ since the first two terms in the expression (14) give a constant contribution to the energy of all states. Although this contribution is dominant according to our earlier assumptions, the spectrum generating perturbation of the hamiltonian is confined to the last two terms and depends solely on the coefficients $b$ and $c$ which are related to the original interactions $\nu_{F}$ according to

$$
b=\frac{1}{70}\left(-7 \nu_{0}+10 \nu_{2}-3 \nu_{4}\right), \quad c=\frac{1}{14}\left(-\nu_{2}+\nu_{4}\right),
$$




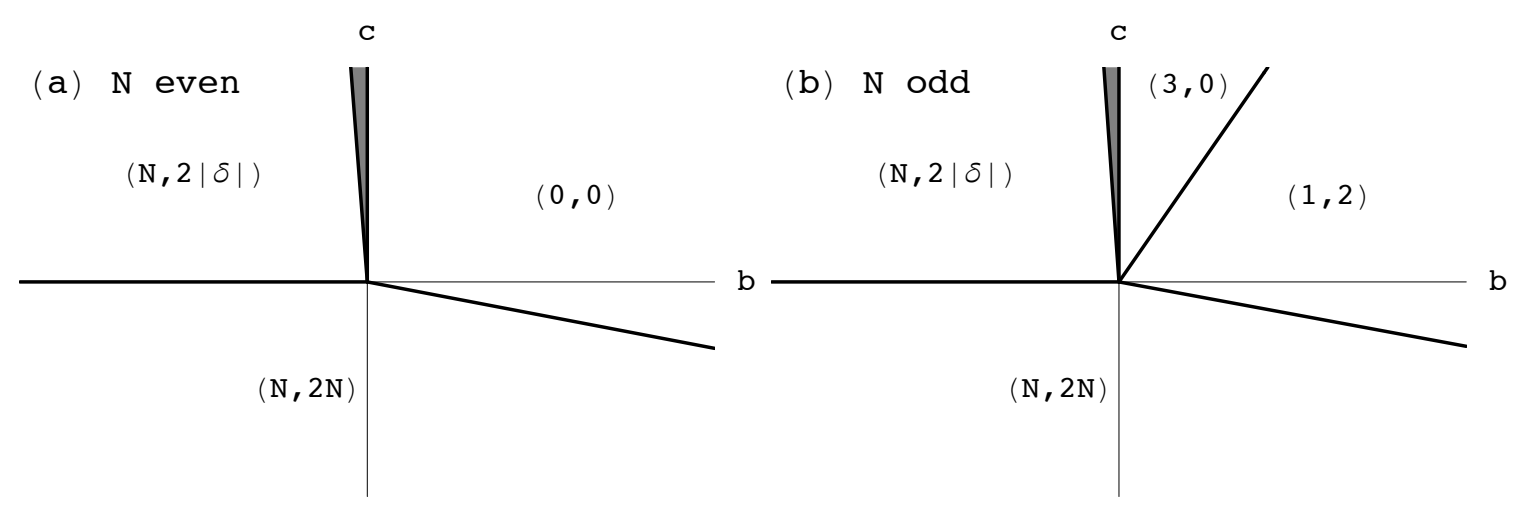

Figure 1. Diagrams of the different phases of a Bose-Einstein condensate of atoms with spin $f=2$ characterized by a ground state $\left(v_{0}, F_{0}\right)$ where $v_{0}$ is the seniority of the ground state and $F_{0}$ is its total spin. The total number of atoms $N$ is even in (a) and odd in (b). The grey area corresponds to a ground state with $\left(v_{0}, F_{0}\right)=(N-3+\delta, 0)$ which only occurs for $\delta= \pm 1$ and disappears in the limit $N \rightarrow \infty$.

The following exact finite- $N$ results are found where the ground state of the condensate is characterized by a seniority $v_{0}$ and a total spin $F_{0}$.

(i) $N$ is even. We introduce $N=6 k+2 \delta$ with $k$ integer and $\delta=-1,0,+1$. The possible ground-state configurations have $\left(v_{0}, F_{0}\right)=(0,0),(N, 2 N),(N, 2|\delta|)$, or $(N-3+\delta, 0)$, the latter existing only for $\delta= \pm 1$,

(ii) $N$ is odd. We introduce $N=6 k+3+2 \delta$ with $k$ integer and $\delta=-1,0,+1$. The possible ground-state configurations have $\left(v_{0}, F_{0}\right)=(1,2),(3,0),(N, 2 N),(N, 2|\delta|)$, or $(N-3+\delta, 0)$, the latter existing only for $\delta= \pm 1$.

The phase diagram displays a richer structure than in the $f=1$ case as is shown in Fig. 1. We observe first of all the presence of the aligned phase where the seniority is maximal, $v_{0}=N$, and all spins are aligned, $F_{0}=2 N$. Secondly, we have a low-seniority (paired) and consequently low-spin phase. For even $N$ this corresponds necessarily to $\left(v_{0}, F_{0}\right)=(0,0)$. For odd $N$ there must be at least one unpaired atom leading to the ground-state configuration $\left(v_{0}, F_{0}\right)=(1,2)$; alternatively, however, it might consist of a triplet of atoms which is coupled to total spin $F_{0}=0$ leading to the ground-state configuration $\left(v_{0}, F_{0}\right)=(3,0)$. The $(1,2)$ and $(3,0)$ phases are divided by the line $b=3 c / 7$. The paired and aligned phases are separated by the line

$$
b=-\frac{2 N(2 N+1)}{N(N+3)} c, \quad b=-\frac{(2 N-2)(2 N+3)}{(N-1)(N+4)} c,
$$

for $N$ even or odd respectively, which in both cases tends to $b=-4 c$ for $N \rightarrow \infty$.

So far we have recovered the aligned and paired phases also encountered for interacting $f=1$ atoms (although the paired phase is somewhat more intricate for $f=2$ due to the possible presence of a triplet of atoms coupled to $F=0$ ). For $f=2$ a third phase occurs for negative $b$ and positive $c$ characterized by high seniority (i.e. unpaired) and low total spin, $\left(v_{0}, F_{0}\right)=(N, 2|\delta|)$. Finally, for $\delta= \pm 1$ there exists a 
pathological region in the phase diagram characterized by $\left(v_{0}, F_{0}\right)=(N-3+\delta, 0)$ (see Fig. (1). It is separated from the high-seniority, low-spin region by the line

$$
b=-\frac{|\delta(\delta+3)|}{4(2 N+\delta)} c,
$$

which tends to $b=0$ for $N \rightarrow \infty$. Hence this region disappears in the large- $N$ limit.

We conclude that the ground state of a BEC consisting of atoms with spin $f=2$ can be of three different types: (i) a maximum-seniority spin-aligned, (ii) a low-seniority low-spin, or (iii) a maximum-seniority low-spin configuration. Note that 'seniority' in this context refers to number of atoms that are not in pairs coupled to $F=0$.

Since the hamiltonian (11) is solvable for $f=2$, all eigenstates, and in particular the three different ground states, can determined analytically. The general expressions given by Chacón et al. [13] reduce to

$$
\begin{aligned}
& |v=N, F=M=2 N\rangle \propto\left(d_{+2}^{\dagger}\right)^{N}|0\rangle, \\
& |v=0, F=M=0\rangle \quad \propto\left(d^{\dagger} \cdot d^{\dagger}\right)^{N / 2}|0\rangle, \\
& |v=N, F=M=0\rangle \quad \propto\left(\left[a^{\dagger} \times a^{\dagger}\right]^{(2)} \cdot a^{\dagger}\right)^{N / 3}|0\rangle,
\end{aligned}
$$

where the $f=2$ atoms are denoted as $d$ bosons. In the second of these expressions it is assumed that $N$ is even and in the third that $N=3 k$; other cases are obtained by adding a single boson or an $F=0$ pair. The $a^{\dagger}$ are the so-called traceless boson operators [13] which are defined as (see also Chapt. 8 of Ref. [14])

$$
a_{m}^{\dagger}=d_{m}^{\dagger}-\frac{d^{\dagger} \cdot d^{\dagger}}{2 N+5} \tilde{d}_{m} .
$$

We emphasize that (18) are the exact finite- $N$ expressions for the eigenstates of the hamiltonian (11). Since in the large- $N$ limit the traceless boson operators $a_{m}^{\dagger}$ become identical to $d_{m}^{\dagger}$, we arrive at a simple interpretation of the three types of configurations: (i) spin-aligned, (ii) condensed into pairs of atoms coupled to $F=0$, and (iii) condensed into triplets of atoms coupled to $F=0$.

How will these features evolve with increasing spin $f$ of the atoms? For arbitrary interaction strengths $\nu_{F}$ in the different $F$ channels the hamiltonian (6) is not solvable. By imposing $f-2$ conditions on $\nu_{F}$ it can be brought into the form (11) and this gives an idea of the structure of the general phase diagram by constructing a two-dimensional slice of it. For example, for atoms with spin $f=3$ the elimination of $a_{1}, a_{2}, b$, and $c$ from Eq. (13) yields the condition $11 \nu_{2}-18 \nu_{4}+7 \nu_{6}=0$. For $f>3$ more conditions on $\nu_{F}$ are found. If all conditions are satisfied, the phase diagram in $b$ and $c$ with

$$
b=\frac{-7 \nu_{0}+10 \nu_{2}-3 \nu_{4}}{14(2 f+1)}, \quad c=\frac{1}{14}\left(-\nu_{2}+\nu_{4}\right),
$$

has properties similar to those in the $f=2$ case. The analysis requires the knowledge of the multiplicity $d_{v}^{(f)}(F)$, (i.e., the number of spin- $f$ atom states with seniority $v$ coupled to total spin $F$ ) which can be derived from the $\mathrm{SO}(2 f+1) \supset \mathrm{SO}(3)$ branching rule [11. We find that for sufficiently large even $N$ there are four competing ground 
states with $\left(v_{0}, F_{0}\right)=(N, f N),(N, 0),(0,0)$, and $(2,2)$, the latter of which disappears as a ground state in the large- $N$ limit. For sufficiently large odd $N$ the four competing ground states have $\left(v_{0}, F_{0}\right)=(N, f N),(N, 0),(1, f)$, and $\left(3, f_{2} \equiv f \bmod 2\right)$, the latter two being separated by the line $b=\left[f(f+1)-f_{2}\left(f_{2}+1\right)\right] c /(4 f+6)$. The results correspond to what is found in the $f=2$ case and lead to an essentially identical $(b, c)$ phase diagram.

Finally, we point out that the appearance of exact seniority ground states requires weaker conditions on $\nu_{F}$ than those that have been discussed so far. In fact, the spinaligned configuration $(N, f N)$ is always an eigenstate of the general hamiltonian (6) because the $F=f N$ state is unique. Furthermore, it can be shown [10] that seniority is a good quantum number if the interaction strengths $\nu_{F}$ satisfy $\lfloor f / 3\rfloor$ conditions only (where $\lfloor x\rfloor$ is the largest integer smaller than or equal to $x$ ). For all cases of any conceivable interest for BECs, this reduces to no condition on the strengths $\nu_{F}$ for $f=1,2$ or just a single one for $f=3,4,5$. So there is at most a single condition required for all eigenstates to carry exact seniority and for the results of this paper to be valid. Nevertheless, the determination of the complete phase diagram for $f>2$ with unconstrained interaction strengths $\nu_{F}$ remains a problem worthy of further investigation.

\section{Acknowledgments}

We acknowledge a conversation with H.T. Stoof that prompted our interest in this problem.

\section{References}

[1] Stamper-Kurn D M, Andrews M R, Chikkatur A P, Inouye S, Miesner H-J, Stenger J and Ketterle W 1998 Phys. Rev. Lett. 802027

[2] Ho T-L, Phys. Rev. Lett. 81, 742 (1998); Ho T-L and Yip S, Phys. Rev. Lett. 82, 247 (1999); Ho T-L and Yin L, Phys. Rev. Lett. 84, 2302 (2000);

[3] C.K. Law, H. Pu, and N.P. BigelowPhys. Rev. Lett. 81, 5257 (1998).

[4] Iachello F and Arima A 1987 The Interacting Boson Model (Cambridge: Cambridge University Press)

[5] Iachello F and Levine R D 1995Algebraic Theory of Molecules(Oxford: Oxford University Press)

[6] Edmonds A R 1957 Angular Momentum in Quantum Mechanics (Princeton: Princeton University Press)

[7] Iachello F 2006 Lie algebras and applications (Berlin: Springer)

[8] Wybourne B G 1974 Classical Groups for Physicists (New York: Wiley-Interscience)

[9] Racah G 1943Phys. Rev. 63367

[10] Talmi I 1993 Simple Models of Complex Nuclei.The Shell Model and Interacting Boson Model(Chur: Harwood)

[11] Gheorghe A and Raduta A A 2004 J. Phys. A 3710951

[12] Chau Huu-Tai P, Frank A, Smirnova N A and Van Isacker P 2002 Phys. Rev. C 66 061302(R)

[13] Chacón E, Moshinsky M and Sharp R T 1976 J. Math. Phys. 17668

[14] Frank A and Van Isacker P 1994 Algebraic Methods in Molecular and Nuclear Structure Physics (New York: Wiley-Interscience) 